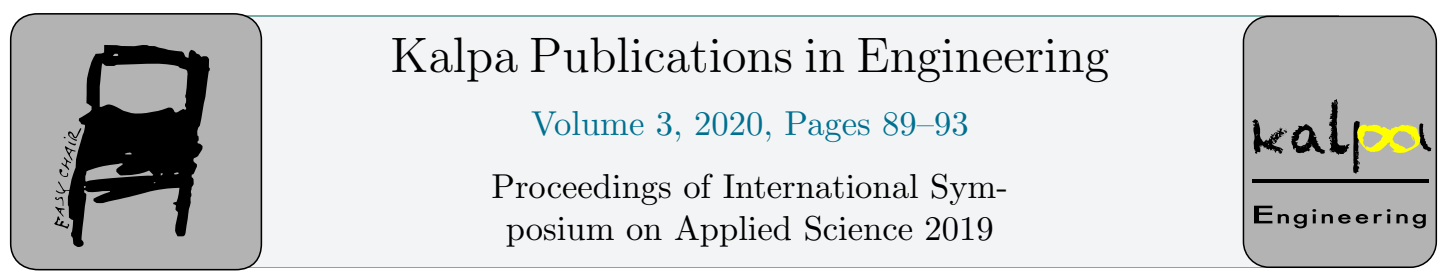

\title{
Extracting The Ionization Rate From Photoelectron Momentum Distribution Induced By Circularly Polarized Laser Pulse
}

\author{
Nguyen Thanh Vinh Pham ${ }^{1}$, Oleg I. Tolstikhin ${ }^{2}$, Toru Morishita ${ }^{3}$ \\ ${ }^{1}$ Department of Physics, Ho Chi Minh City University of Education \\ ${ }^{2}$ Moscow Institute of Physics and Technology, Dolgoprudny 141700, Russia \\ ${ }^{3}$ Institute for Advanced Science, The University of Electro-Communications, Tokyo, Japan \\ vinhpham@hcmup.edu.vn
}

\begin{abstract}
In this paper the adiabatic theory proposed in [6] is implemented to consider the ionization process of molecular system exposed to circularly polarized laser pulse. The photoelectron momentum distribution exhibits strong evidence of the properties of molecular orbital. We also present the possibility to retrieve the ionization rate of ionized electron from photoelectron momentum distribution in the transverse plane respecting to the polarized plane of the laser field. This results are vital in the viewpoint of experiment. Several states of molecular hydrogen ion are considered for illustration.
\end{abstract}

\section{Introduction}

Extracting target structure and dynamics information from high-order harmonic spectra and photoelectron momentum distributions (PEMDs) generated in the ionization of atoms and molecules by intense low-frequency laser pulses is one of the fundamental goals of strong field physics. A powerful technique of tomographic imaging of molecular orbitals is established in high-order harmonic spectroscopy [1]. Photoelectron spectroscopy suggests several complementing approaches of two kinds, depending on whether rescattered or direct photoelectrons perform imaging. Rescattered photoelectrons recollide with the parent ion before arriving at a detector [2], so their contribution to strong-field PEMDs bears information on the target collisional properties. One widely used approach in rescattering photoelectron spectroscopy focuses on the high-energy part of PEMDs dominated by nearly backward rescattered photoelectrons [3]. The contribution of direct photoelectrons to PEMDs contains information on the tunneling ionization process and, through this, on the ionizing orbital. While in rescattering photoelectron spectroscopy linearly polarized laser pulses are usually used, to force a photoelectron to return to the parent ion for recollision, for the observation of direct photoelectrons it is preferable to use circularly polarized pulses to eliminate rescattering [4] which contaminates 
tunneling observables. Thus, circular polarization is essential for accurate measurements of tunneling ionization rates of molecules [5]. In this report, we introduce the possibility to extract the ionization rates from the PEMDs induced by circularly polarized laser pulse based on the adiabatic theory [6,7]. The molecular hydrogen ion in different states is used for illustration.

\section{Theory}

The TDSE in the dipole approximation and length gauge reads (atomic units are used in the paper)

$$
i \frac{\partial \psi(\mathbf{r}, t)}{\partial t}=\left[-\frac{1}{2} \Delta+V(\mathbf{r})+\mathbf{F}(t) \mathbf{r}\right] \psi(\mathbf{r}, t),
$$

where the potential $V(\mathbf{r})$ describes the interaction of the active electron with the parent ion and $\mathbf{F}(t)$ is the electric field of the pulse. The field is presented in the form $\mathbf{F}(t)=F(t) \mathbf{e}(t)$, where $F(t) \square 0$ is the field strength and $\mathbf{e}(t)$ is the polarization vector satisfying $\mathbf{e}^{2}(t)=1$. In this paper, we consider pulses with a Gaussian envelope and propagates along the $z$ axis and circularly polarized in the $(x, y)$ plane. The initial condition for $\mathrm{Eq}(1)$ is

$$
\psi(\mathbf{r}, t \rightarrow-\infty)=\phi_{0}(\mathbf{r}) e^{-i E_{0} t}
$$

where $E_{0}$ and $\emptyset_{o} r$ are the energy and wave function of a bound state of the unperturbed system. The main observable of interest here is the PEMD defined by [6]

$$
P(\mathbf{k})=|I(\mathbf{k})|^{2}, \quad I(\mathbf{k})=\left\langle\psi_{\mathbf{k}}^{(-)} \mid \psi(t \rightarrow \infty)\right\rangle .
$$

Within the adiabatic theory, the ionization amplitude is obtained in the form $I(\mathbf{k})=I_{a}(\mathbf{k})+I_{r}(\mathbf{k})$ , where the adiabatic $I_{a}(\mathbf{k})$ and rescattering $I_{r}(\mathbf{k})$ parts represent contributions from electrons which go directly to a detector after their release from the system and those which experience rescattering before arriving at a detector, respectively. Under the influence of circularly polarized laser pulses, the possibility of recollision between ionized electron and its parent ion is eliminated. Thus the leadingorder term of $I_{a}(\mathbf{k})$ is given by [6]

Here, the quantum action is

$$
I_{a}(\mathbf{k})=e^{i \pi / 4}(2 \pi)^{1 / 2} \sum_{i} \frac{A\left(\Delta \mathbf{k}_{\perp} ; t_{i}\right)}{F^{1 / 2}\left(t_{i}\right)} \times \exp \left[i S\left(t_{i}, \mathbf{k}\right)-i s\left(t_{i}\right)\right] .
$$

$$
s(t)=E_{0} t+\int_{-\infty}^{t}\left[E\left(t^{\prime}\right)-E_{0}\right] d t^{\prime}
$$

and the classical action is

$$
S(t, \mathbf{k})=\frac{1}{2} \mathbf{k}^{2} t-\frac{1}{2} \int_{t}^{\infty}\left[\mathbf{u}_{i}^{2}\left(t^{\prime}, \mathbf{k}\right)-\mathbf{k}^{2}\right] d t^{\prime},
$$

where

and

$$
\mathbf{u}_{i}^{2}(t, \mathbf{k})=\mathbf{k}-\mathbf{k}_{a}(t)
$$

$$
\mathbf{k}_{a}(t)=\mathbf{v}_{\infty}-\mathbf{v}(t){ }_{(8)}
$$

The vector $\mathbf{u}_{i}(t, \mathbf{k})$ gives the initial velocity with which an electron driven by the field should start its motion at time $t$ to have the final velocity after the end of the pulse equal to $\mathbf{k}$. The moments of ionization are defined by

$$
\mathbf{e}(t) \mathbf{u}_{i}(t, \mathbf{k})=0 \rightarrow t=t_{i}(\mathbf{k})
$$




\section{Result}

We apply the adiabatic theory to the analysis of PEMDs of the hydrogen molecular ion whose geometry is illustrated in Fig. 1. The nuclei are assumed to be located on the $\mathrm{x}$ axis in the polarization plane, symmetrically with respect to the origin. The potential describing the interaction between the electron and the nuclei is given as

$$
\mathrm{V}(\mathbf{r})=-\frac{1}{\sqrt{|\mathbf{r}-\mathbf{R} / 2|^{2}+a^{2}}}-\frac{1}{\sqrt{|\mathbf{r}+\mathbf{R} / 2|^{2}+a^{2}}}
$$

where $\mathbf{R}=(R, 0,0), R=2$ and the softening parameter is $a=0.3$.

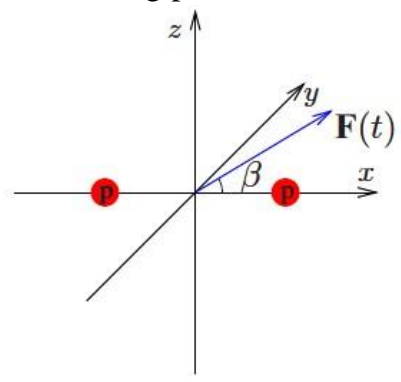

Figure 1: Geometry of $\mathrm{H} 2 \square$ exposed to the laser pulse.

In this case, the ionization rate of the system is extracted from the PEMD in the plane perpendicular to the polarized plane of the laser field and show here the extraction for $1 \mathrm{~s} \sigma$ state in the Fig. 2 which presents the dependece of ionization rate on the orientation angle. The parameters of laser fields are $\mathrm{F}=0.1,15$ cycle, and $\mathrm{T}=1800(\omega \approx 0.052)$. The extraction from PEMD is the red dot which quantitatively consistence to the exact calculation based on

Siegert state for static field as solid black curve [8]. The behavior of this dependence can be qualitatively described by approximation theory

[9].

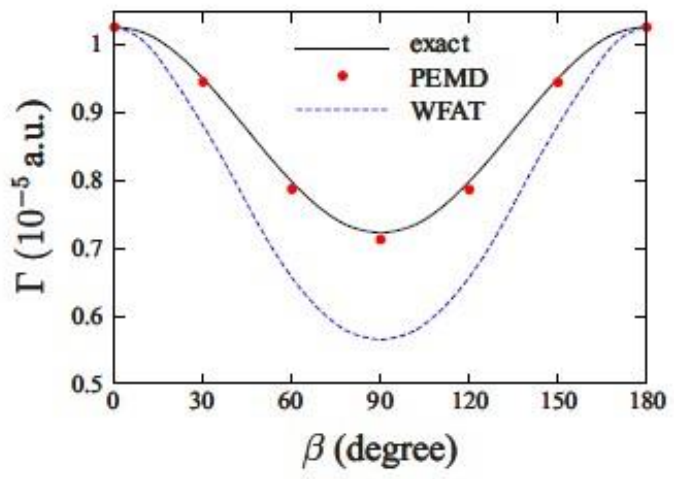

Figure 2: Ionization rate of $1 \mathrm{~s} \square$ state as a function of orientation angle.

For $2 \mathrm{p} \sigma$ state, the result of extration is shown in the Fig. 3 which has maximum at $\beta=0^{0}$ and minimum at $\beta=90^{\circ}$. Note that at $\beta=90^{\circ}$, the extraction and exact ionization rates are different from zero due to the contribution of next-to-dominant channel. Here the parameters of laser are similar to that in Fig. 1 except 


$$
F=0.03
$$

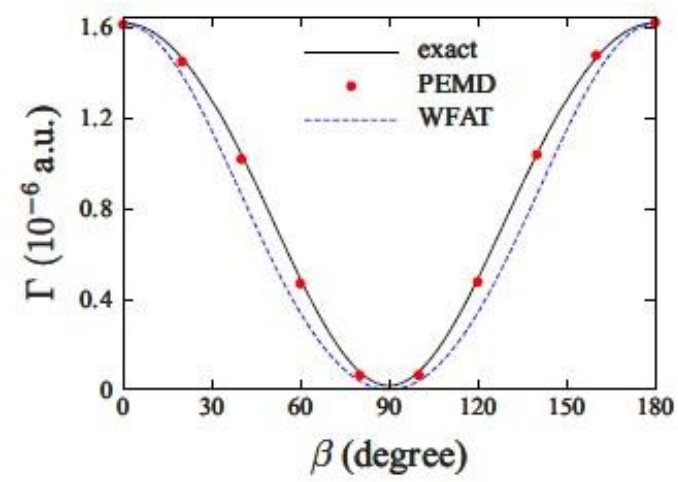

Figure 3: Ionization rate of $2 \mathrm{p} \square$ state as a function of orientation angle.

Finally, the result for $2 \mathrm{p} \sigma$ state is shown in Fig.4. Obviously, in case of $2 \mathrm{p} \sigma$ state, the prediction of WFAT is not qualified due to the lack of several next-to-dominant channels. This feature emphasizes the important role of exact numerical calculation.

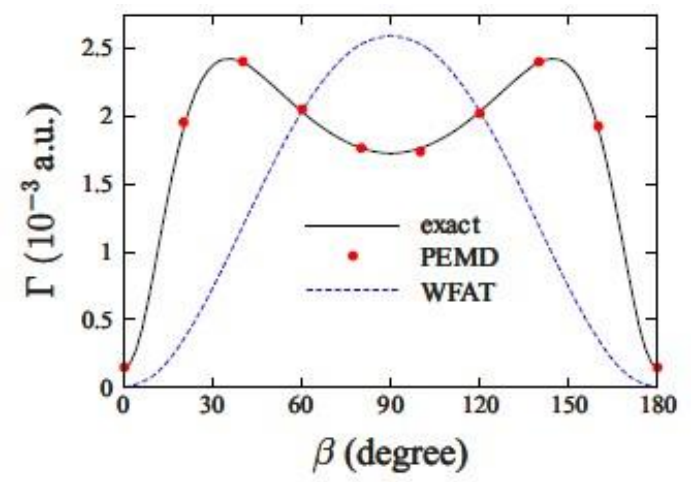

Figure 4: Ionization rate of $2 \mathrm{p} \square$ state as a function of orientation angle.

\section{Conclusion}

In this paper, we have shown that the ionization rate can be imprinted in the transverse plane of PEMD and is straightforwardly extracted. To validation, the extractions are compared to the exact calculation using Siegert state in static field for. The numerical results are also described by WFAT. We hope that this result is going to be vital for the sake of experiment since the measurement of PEMD induced by circularly polarized laser field is much easier than by static field, especially for strong field.

\section{References}

F. Krausz and M. Ivanov, Rev. Mod. Phys. 81, 163 (2009).

P. B. Corkum, Phys. Rev. Lett. 71, 1994 (1993). 
G. G. Paulus, W. Becker, W. Nicklich, and H. Walther, J. Phys. B: At. Mol. Opt. Phys. 27, L703 (1994).

H. Niikura, F. Légaré, R. Hasbani, A. D. Bandrauk, M. Yu. Ivanov, D. M. Villeneuve, and P. B. Corkum, Nature (London) 417, 917 (2002).

D. Pavicic, K. F. Lee, D. M. Rayner, P. B. Corkum, and D. M. Villeneuve, Phys. Rev. Lett. 98, 243001 (2007).

O. I. Tolstikhin and T. Morishita, Phys. Rev. A 86, 043417 (2012).

V. N. T. Pham, O. I. Tolstikhin and T. Morishita, Phys. Rev. A 99, 013428 (2019).

V. N. T. Pham, O. I. Tolstikhin and T. Morishita, Phys. Rev. A 89, 033426 (2014).

O. I. Tolstikhin, T. Morishita, and L. B. Madsen, Phys. Rev. A 84, 053423 (2011). 